\title{
Spatially Distinct Functional Output Regions within the Central Nucleus of the Inferior Colliculus: Implications for an Auditory Midbrain Implant
}

\author{
Hubert H. Lim and David J. Anderson \\ Kresge Hearing Research Institute, Department of Biomedical Engineering, and Department of Electrical Engineering and Computer Science, University of \\ Michigan, Ann Arbor, Michigan 48109
}

\begin{abstract}
The inferior colliculus central nucleus (ICC) has potential as a new site for an auditory prosthesis [i.e., auditory midbrain implant (AMI)] for deaf patients who cannot benefit from cochlear implants (CIs). We have previously shown that ICC stimulation achieves lower thresholds, greater dynamic ranges, and more localized, frequency-specific primary auditory cortex (A1) activation than CI stimulation. However, we also observed that stimulation location along the caudorostral (isofrequency) dimension of the ICC affects thresholds and frequency specificity in A1, suggesting possible differences in functional (output) organization within the ICC. In this study, we electrically stimulated different regions along the isofrequency laminas of the ICC and recorded the corresponding A1 activity in ketamineanesthetized guinea pigs using multisite probes to systematically assess ICC stimulation location effects. Our results indicate that stimulation of more rostral and somewhat ventral regions within an ICC lamina achieves lower thresholds, smaller discriminable level steps, and larger evoked potentials in A1. We also observed longer first spike latencies, which correlated with reduced spiking precision, when stimulating in more caudal and dorsal ICC regions. These findings suggest that at least two spatially distinct functional output regions exist along an ICC lamina: a caudal-dorsal region and a rostral-ventral region. The AMI will be implanted along the tonotopic axis of the ICC to achieve frequency-specific activation. However, stimulation location along the ICC laminas affects response properties that have shown to be important for speech perception performance, and needs to be considered when implanting future AMI patients.
\end{abstract}

Key words: auditory cortex; auditory midbrain implant; auditory pathways; deep brain stimulation; inferior colliculus; isofrequency

\section{Introduction}

Although cochlear implants (CIs) can restore intelligible speech perception in patients with sensorineural hearing loss (Rauschecker and Shannon, 2002; Skinner et al., 2002), they are ineffective for patients with severely damaged auditory nerves or unimplantable cochleas. An alternative is the auditory brainstem implant (ABI). However, placement of the ABI is difficult and it has achieved limited performance levels (comparable with singlechannel CIs) in its main target group of neurofibromatosis type II (NF2) patients (Shannon et al., 1993; Lenarz et al., 2001; Otto et al., 2002). Previously, we demonstrated in a guinea pig model that the inferior colliculus central nucleus (ICC) has potential as a new site for an auditory prosthesis (Lim and Anderson, 2003, 2006; M. Lenarz et al., 2006). In particular, ICC stimulation

\footnotetext{
Received Nov. 27, 2006; revised June 1, 2007; accepted June 25, 2007.

This work was supported by National Institutes of Health (NIH) Grants P41 EB2030, P30 DC05188, T32 DC00011, and F31 DC007009 and by the University of Michigan Center for Wireless Integrated MicroSystems (National Science Foundation Engineering Research (enter). Multisite probes were provided by the University of Michigan Center for Neural Communication Technology (NIH Grant P41 EB2030). We thank James Wiler, Jamille Hetke, Chris Ellinger, Jack Briggs, and Dwayne Vailliencourt for surgical and technical support. We also thank Drs. Bryan Pfingst, John Middlebrooks, Nell Cant, and Daryl Kipke for serving on H.H.L.'s dissertation committee and providing suggestions on a previous version of this manuscript.

Correspondence should be addressed to Dr. David J. Anderson, Department of Electrical Engineering and Computer Science, University of Michigan, 1301 Beal Avenue, Ann Arbor, Ml 48109-2122. E-mail: dja@umich.edu.

DOI:10.1523/JNEUROSCI.5127-06.2007

Copyright $\odot 2007$ Society for Neuroscience $\quad$ 0270-6474/07/278733-11\$15.00/0
}

achieves lower thresholds, greater dynamic ranges, and more localized, frequency-specific primary auditory cortex (A1) activation than CI stimulation. This suggests that an auditory midbrain implant array (AMI) aligned along the tonotopic axis of the ICC may enhance both level (Zeng and Galvin, 1999; Loizou et al., 2000) and frequency (Friesen et al., 2001; Shannon et al., 2004) coding with less energy requirements compared with the CI. However, we also observed that stimulation location along the caudorostral (isofrequency) dimension of the ICC affects thresholds and frequency specificity in A1.

Anatomical studies have shown that spatially distinct functional zones exist within the ICC (Roth et al., 1978; BrunsoBechtold et al., 1981; Shneiderman and Henkel, 1987; Oliver et al., 1997; Cant and Benson, 2003; Loftus et al., 2004; Cant and Benson, 2006). Furthermore, electrophysiological studies have demonstrated that different regions along the isofrequency ICC laminas process sound information (e.g., threshold, frequency tuning, frequency sweep speed, best modulation frequency, latency, binaurality) in different ways (Semple and Aitkin, 1979; Stiebler, 1986; Langner and Schreiner, 1987; Schreiner and Langner, 1988; Ehret, 1997; Hage and Ehret, 2003). Thus, it is hypothesized that stimulation location within the ICC will affect auditory cortical activity. However, it is not yet clear from those studies how the functional zones are organized with respect to output projections to higher auditory structures and, more im- 
portantly for an AMI, how stimulation of different regions along the ICC laminas will affect auditory perception.

Therefore, we systematically investigated the effects of stimulation location along the isofrequency layers of the ICC on thresholds, evoked potential magnitudes, discriminable level steps, and temporal response patterns in A1 of ketamineanesthetized guinea pigs. These neural parameters may correlate with perceptual features important for sound perception. Lower neural thresholds and larger evoked potentials may both provide neural correlates for lower perceptual thresholds. Smaller discriminable level steps should allow for a more accurate representation of the sound envelope and improve speech perception performance (Pfingst et al., 1983; Nelson et al., 1996). Different temporal features, such as temporal precision, have also been shown to be important for speech perception (Shannon et al., 1995). Furthermore, these stimulation location effects can provide insight as to how different regions along the ICC laminas are functionally organized with respect to their output projections.

\section{Materials and Methods}

Basic surgical procedures, and methods for stimulation and recording were similar to those presented in our previous work (Lim and Anderson, $2003,2006,2007)$. For this study, we electrically stimulated different regions within an isofrequency lamina of the ICC (one lamina per animal) and recorded the corresponding neural activity in the main input layer of A1 [one site per animal with a similar best frequency (BF) to the ICC lamina] using multisite probes in anesthetized guinea pigs. Current source density analysis, acoustic-driven responses, and histological techniques were used to confirm that all sites were correctly positioned within the ICC and A1. We then assessed the effects of ICC stimulation location on thresholds, evoked potentials, discriminable level steps, and various temporal features recorded in A1.

\section{Anesthesia and surgery}

We performed experiments on young pigmented guinea pigs (402-448 g; Elm Hill Breeding Labs, Chelmsford, MA) in accordance with policies of the University of Michigan Committee on the Use and Care of Animals. The animals were initially anesthetized with an intramuscular injection of ketamine hydrochloride $(40 \mathrm{mg} / \mathrm{kg}$ ) and xylazine $(5 \mathrm{mg} / \mathrm{kg}$ ) with additional supplements to maintain an areflexive state. Siliconsubstrate, thin-film, multisite Michigan probes (Center for Neural Communication Technology, University of Michigan, Ann Arbor, MI) (Drake et al., 1988; Anderson et al., 1989) were then stereotaxically inserted into the ICC and Al (Fig. 1). The ICC probe had two shanks separated by $500 \mu \mathrm{m}$ (all distances are center to center) where each shank had eight electrode sites linearly spaced at intervals of $200 \mu \mathrm{m}$. Each shank was $15 \mu \mathrm{m}$ thick, $8 \mathrm{~mm}$ long, and tapered in width from $400 \mu \mathrm{m}$ to a few micrometers at the tip. Each site had an area of $\sim 400 \mu \mathrm{m}^{2}$ and was activated to an impedance of $<100 \mathrm{k} \Omega$. Activating the ICC sites from iridium to iridium oxide (via cyclic voltammetry) enabled us to use them for both recording and stimulation (Anderson et al., 1989). However, based on the electrochemical charge injection limit for iridium oxide $(\sim 3$ $\mathrm{mC} / \mathrm{cm}^{2}$ ) (Beebe and Rose, 1988; Weiland and Anderson, 2000), we limited stimulation to a maximum current level of $56.2 \mu \mathrm{A}(200 \mu \mathrm{s} / \mathrm{phase}$ pulses). We presented stimulus levels in logarithmic (decibel) steps relative to $1 \mu \mathrm{A}$. Thus, $56.2 \mu \mathrm{A}$ corresponded to the maximum stimulus level $(35 \mathrm{~dB})$ still within the charge injection limit of $60 \mu \mathrm{A}$. The ICC probe was inserted $45^{\circ}$ off the sagittal plane through the occipital cortex (not shown in Fig. 1) into the inferior colliculus (IC) where each shank was aligned along the tonotopic axis of the ICC and each site was placed into a different frequency region. The A1 probe consisted of a single shank with 16 sites linearly spaced at intervals of $150 \mu \mathrm{m}$. Each shank was 15 $\mu \mathrm{m}$ thick, $5 \mathrm{~mm}$ long, and tapered in width from $200 \mu \mathrm{m}$ to a few micrometers at the tip. Each site had an area of $\sim 400 \mu \mathrm{m}^{2}$ and impedance (unactivated) of $1-2 \mathrm{M} \Omega$. The $\mathrm{A} 1$ probe was inserted approximately perpendicular to the cortical surface along a BF column of A1 (Abeles and Goldstein, 1970; Redies et al., 1989; Wallace et al., 2000).

For each animal, we inserted the Al probe into only one BF column and performed current source density (CSD) analysis to determine which site was located in the main input layer of A1 (for more details, see below, Placement of probes). We then inserted the ICC probe into several different locations along the isofrequency dimension of the ICC where for each placement one site with a similar BF to that of the Al site was used for electrical stimulation. After each probe placement, the brain was covered with agarose to reduce swelling, pulsations, and drying.

\section{Stimulation and recording setup}

Experiments were conducted in a sound-attenuating chamber and controlled by a computer interfaced with TDT hardware (Tucker-Davis Technology, Alachua, FL) using custom software written in MatLab script (Mathworks, Natick, MA). To aid in the positioning of the probes, we presented varying levels of pure tones and broadband noise (all $50 \mathrm{~ms}$ in duration) to elicit acoustic-driven activity in the ICC and A1. All neural signals were passed through analog DC-blocking and antialiasing filters from $1.6 \mathrm{~Hz}$ to $7.5 \mathrm{kHz}$. For spike analysis, these signals were then digitally filtered between 0.3 and $3 \mathrm{kHz}$. After placement of the probes, we connected the ICC probe to a custom-made optically isolated current stimulator for electrical stimulation. Each ICC site was stimulated as an active electrode where the return was through a wire positioned in a neck muscle (monopolar configuration). Electrical stimuli consisted of single biphasic, charge-balanced pulses (200 $\mu$ s/phase, cathodic-leading) from 1 to $56.2 \mu \mathrm{A}$ in decibel steps. Forty trials of each stimulus at $2 / \mathrm{s}$ were presented in a randomized sequence across all sites and levels to reduce adaptive effects. Neural data were recorded on all $16 \mathrm{~A} 1$ sites in response to stimulation of each ICC site. The recording ground wire was positioned under the skin $\sim 2 \mathrm{~cm}$ rostral to bregma.

\section{Placement of probes}

Poststimulus time histograms (PSTHs) and frequency response maps (FRMs) were plotted to confirm that probes were correctly positioned along the tonotopic axis of the ICC and along a BF column in A1. Details on PSTH, FRM, and BF calculation are presented in a previous publication (Lim and Anderson, 2006). In the ICC, we typically observed sustained PSTHs in response to broadband noise and, as shown in Figure 
A

ICC FRMs
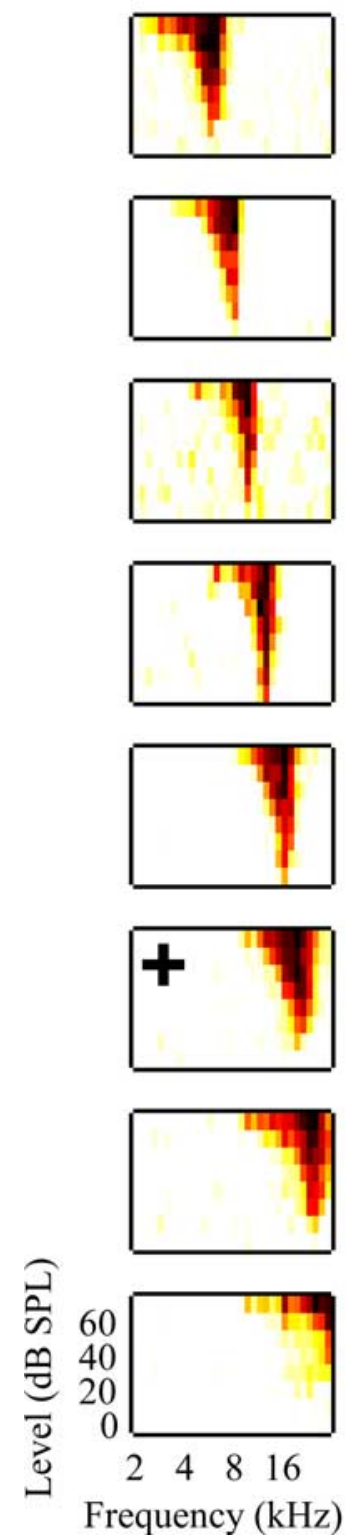

Figure 2. A, FRMs recorded on eight different sites linearly spaced (200 $\mu \mathrm{m}$ apart) along the tonotopic gradient of the ICC for one ICC probe shank. $B$, FRMs recorded on eight different sites linearly spaced ( $150 \mu \mathrm{m}$ apart) along a $\mathrm{BF}(\sim 21 \mathrm{kHz})$ column in A1 for the A1 probe. Only FRMs for 8 of the 16 sites along the $A 1$ probe that were located approximately within layers II through $V$ were plotted for this example. The asterisk corresponds to the main input layer site in A1 (for more details, see Materials and Methods, Placement of probes). The plus sign corresponds to the one selected ICC site that was BF aligned with the selected $A 1$ site. For each FRM, the abscissa is frequency (2-30 kHz; 8 steps/octave) and the ordinate is stimulus intensity [0-70 dB sound pressure level (SPL); $10 \mathrm{~dB}$ steps]. The color scale corresponds to normalized driven spike rate where all negative FRM values were set to zero to improve visualization.

$2 \mathrm{~A}$, FRMs that exhibited an orderly shift in BF (Snyder et al., 2004). In A1, we first recorded surface potentials in response to pure tones using the tip site of our probe and determined the borders of A1 based on its BF organization (Wallace et al., 2000). We then inserted and aligned the probe along a specific BF column. Obtaining FRMs with approximately similar BFs, as shown in Figure $2 B$, confirmed that our probe was correctly positioned in $\mathrm{A} 1$.

To identify the recording site along our A1 probe that was located in

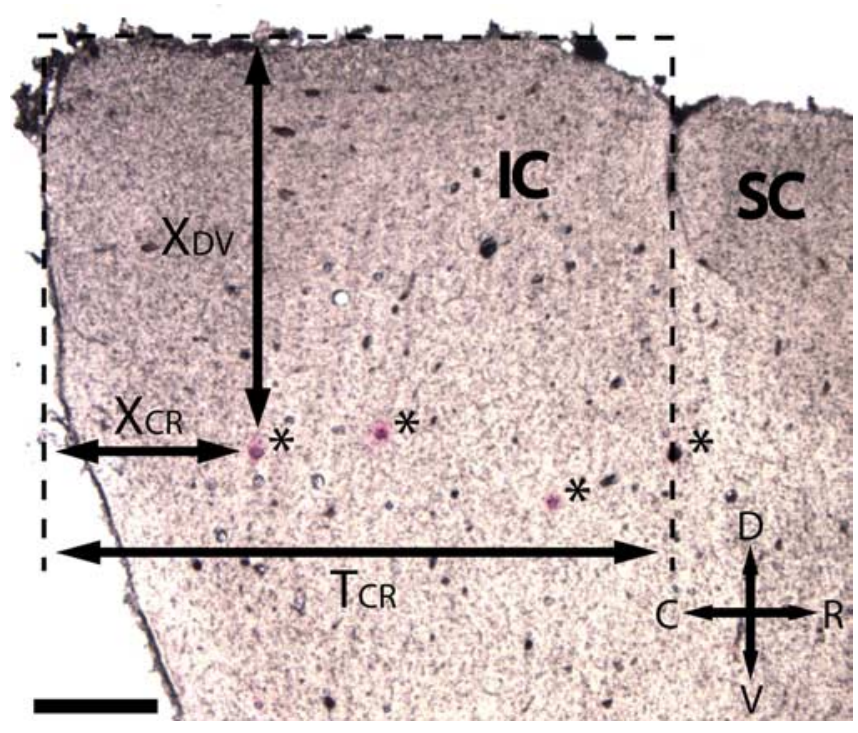

Figure 3. Sagittal section of the $\mathrm{IC}$ corresponding to a normalized distance of 0.31 from the lateral edge of the IC to the midline of the brain. The ICC probe, which had two shanks, was inserted $45^{\circ}$ off the sagittal plane. Thus, the stained shank tracts for two probe placements appear as red dots (asterisks) in the sagittal sections. The distances of the red dots from the dorsal $\left(X_{D V}\right)$ and caudal $\left(X_{C R}\right)$ edges of the IC were measured and appropriately normalized to provide dimensionless values for the ICC shank locations across animals. One site from each shank placement was positioned within the target best frequency lamina. Thus, the dimensionless shank locations indirectly correspond to the relative site locations within an ICC lamina. The most rostral dot was located outside the ICC based on acoustic properties and excluded from analysis. For more details, see Materials and Methods, ICC probe histology. Scale bar, $0.5 \mathrm{~mm}$. C, Caudal; D, dorsal; $R$, rostral; $V$, ventral.

the main input layer of A1, we performed CSD analysis (Muller-Preuss and Mitzdorf, 1984; Mitzdorf, 1985). The one-dimensional CSD approximation provides a consistent representation for the current sinks and sources associated with columnar synaptic activity in the auditory cortex. The main input layer of A1, which approximately corresponds to layer III/IV (Huang and Winer, 2000; Smith and Populin, 2001), was taken as the site with the shortest latency current sink and PSTH response. Details on and results using this method have been presented previously (Lim and Anderson, 2007).

\section{ICC probe histology}

To identify the location of each probe shank within the ICC sections, we dipped the probe into a red stain [ $3 \mathrm{mg}$ of Di-I (1,1-dioctadecyl-3,3,3', $3^{\prime}$ tetramethylindocarbocyanine perchlorate) per $100 \mu \mathrm{l}$ of acetone; Invitrogen, Eugene, OR] before each insertion into the brain. An effective protocol consisted of repeatedly (10 times) dipping the probe into the stain for $10 \mathrm{~s}$ and letting it dry in air for $10 \mathrm{~s}$ (DiCarlo et al., 1996). At the end of each experiment, the animal was decapitated and the head was immersed into $10 \%$ formaldehyde for $\sim 5 \mathrm{~d}$. The brain was removed and placed in $20 \%$ sucrose solution until the tissue sank. Frozen sections ( 40 $\mu \mathrm{m}$ thick) were cut in the sagittal plane. The sections were mounted onto slides and examined through a microscope, and images of each section were saved for additional analysis.

Figure 3 shows an example of a sagittal section with four red dots (labeled with asterisks). Because the ICC probe was inserted $45^{\circ}$ off the sagittal plane, each shank tract appeared as a dot in the sagittal section. Once the dots were located, we were able to measure their distances from the caudal $\left(X_{\mathrm{CR}}\right)$ and dorsal $\left(X_{\mathrm{DV}}\right)$ edge of the IC. Along the caudorostral dimension, we normalized $X_{\mathrm{CR}}$ by $T_{\mathrm{CR}}$, which was the total distance between the caudal edge and the IC-superior colliculus (SC) division. There were no consistent landmarks along the dorsoventral dimension. However, because the animals were similar in size, we normalized $X_{\mathrm{DV}}$ by the maximum dorsoventral shank location still within the ICC across all our sites and animals. This maximum value approximately corresponded 
to the ventral edge of the ICC because all other sites (across all animals) placed more ventrally did not exhibit acoustic-driven responses characteristic of ICC neurons as described in the previous section. Because these parameters change along the mediolateral dimension (for different sagittal sections), these measurements were made only on the sagittal section corresponding to a normalized distance of 0.31 from the lateral edge of the IC to the midline of the brain. Normalizing these distances provided dimensionless coordinates within the ICC to pool our data across animals. For analysis, only sites confirmed to be within the ICC based on acoustic-driven responses were used. The most rostral dot in Figure 3 is an example of a shank that was located outside the ICC and excluded from analysis. Although we measured the dorsoventral distance (from the dorsal edge of the IC) of each shank placement (red dot), all the stimulated sites for a given animal were located within a similar BF lamina, which is approximately oriented $45^{\circ}$ off the dorsoventral axis as shown in Figure 1 A (Merzenich and Reid, 1974; Malmierca et al., 1995; Schreiner and Langner, 1997; Snyder et al., 2004). Thus, the dorsoventral distance provides an approximately scaled value [by a $\cos \left(45^{\circ}\right)$ factor] for the dorsomedial-to-ventrolateral location (isofrequency dimension). Throughout the paper, we will use the terms "dorsal" and "ventral" to be consistent with our histological measurements from Figure 3 but to indirectly represent the location along an ICC lamina.

One limitation in our normalization procedure is that the different isofrequency laminas do not exhibit the same dimensions nor are they positioned in the same relative location from the caudal and dorsal edges of the IC (Malmierca et al., 1996). Furthermore, slight variations in tissue preparation and ICC probe orientations across animals could further introduce location errors. Thus, we cannot guarantee that the normalized coordinates $\left(X_{\mathrm{CR}}, X_{\mathrm{DV}}\right)$ in one lamina corresponds to the same relative location in another lamina with the same coordinates. Because we needed to pool data across laminas because of the limited number of sites per lamina for analysis, these spatial misalignments may have added additional variability that masked some systematic stimulation location effects on different A1 response properties. We can only claim that the stimulation location effects observed in this study are a few of possibly many that occur along the isofrequency dimension of the ICC.

\section{Data analysis}

For acoustic stimulation, we analyzed the neural activity (multiunit and evoked potentials) recorded on all sites for both the ICC and A1 probes to aid in their placement in each animal. For recordings in $\mathrm{A} 1$ in response to ICC stimulation (single pulses), we only used the neural activity recorded on one site centered in the main input layer of A1. This activity was recorded for stimulation of different sites throughout an isofrequency lamina of the ICC with a similar BF to that of the Al site. Only one BF lamina was investigated for each animal. Methods for spike detection and elimination of ICC stimulation artifacts (i.e., electrical artifacts, antidromic spikes) are similar to those used in previous studies (Lim and Anderson, 2006, 2007). Below, we present the methods for calculating the different parameters used in this paper.

Electrical threshold. We used a method based on a signal detection theory (SDT) (Green and Swets, 1966; Britten et al., 1992). We plotted receiver operating characteristic (ROC) curves from trial-by-trial spike rates for the "stimulus" and the "no-stimulus" case for different stimulation levels. By adjusting a criterion spike rate level, we were able to obtain the percentage of "stimulus" trials exceeding that criterion (correct hits) and that of "no-stimulus" trials (false alarms) and plotted these values for varying criterion levels to obtain an ROC curve. Spike rates were computed from a window 2-30 ms after stimulus onset or, in the case of no-stimulus, a $28 \mathrm{~ms}$ window from the spontaneous data. This time window was selected based on visual inspection of all the PSTH responses and where we consistently observed most of the stimulusdriven response. Based on this SDT framework, the area under the ROC curve corresponded to the performance level [or percentage correct (PC)] of an ideal observer detecting a stimulus based on the measured spike rates in a two-alternative-force-choice paradigm. After computing the area of each ROC curve, we were able to plot PC as a function of stimulus level (neurometric curves) that ranged from 0.5 (chance) to 1 (perfect detection). These curves were interpolated for analysis. For this

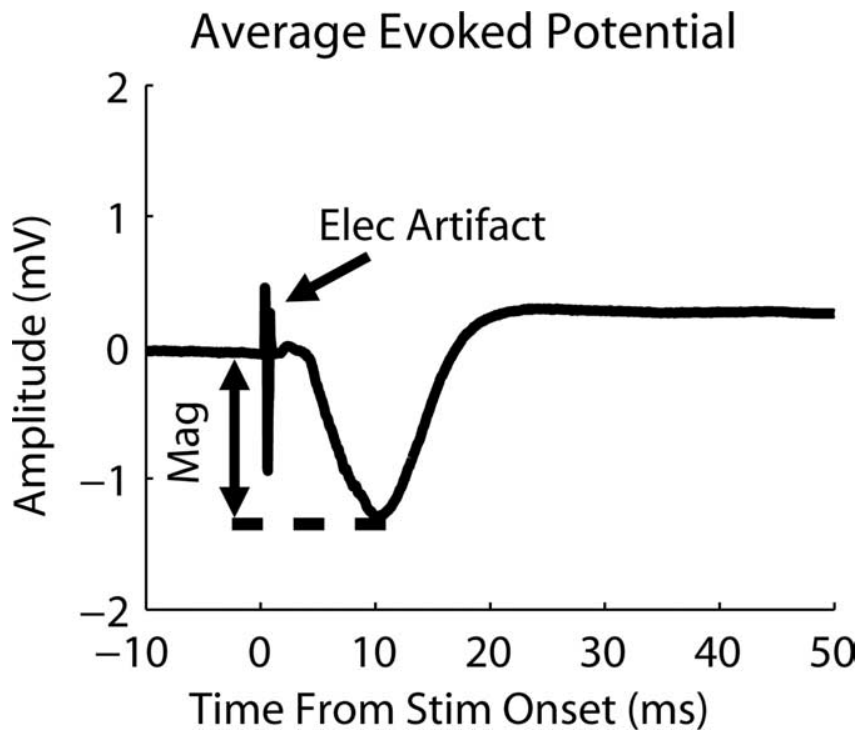

Figure 4. Average evoked potential recorded on the main input layer site in $A 1$ in response to stimulation (Stim) (28.2 $\mu \mathrm{A} ; 40$ trials) of an ICC site with a similar best frequency ( $21 \mathrm{kHz})$. The electrical artifact (Elec artifact) and magnitude (Mag) for the response are labeled.

SDT method, electric threshold was defined as the stimulus level corresponding to a PC equal to 0.76 . This value was selected because it corresponds to a discrimination index $\left(d^{\prime}\right)$ equal to 1 if we assume that the trial-by-trial spike rates for the "stimulus" and "no-stimulus" case follow equal-variance normal distributions. Throughout this paper, we will use $d^{\prime}$ instead of PC because it is a monotonic function of $\mathrm{PC}$ and provides a more commonly used measure in psychophysics. This SDT procedure gives a conservative estimate of threshold in that it estimates the level at which a stimulus-driven response can be detected on single trials. Often, an increase in across-trial mean spike rate could be observed at current levels a few decibels lower than the SDT-derived threshold (for examples, see Fig. $5 A, B)$.

Discriminable level steps. A discriminable level step corresponds to the change in ICC current level (microampere or decibel) needed to elicit a just noticeable difference (JND) in spike rate activity in Al above threshold. This JND level step was used as a measure for assessing what the perceptual level discrimination limens may be in response to ICC stimulation. To calculate the JND level steps, we applied a similar method to that used for SDT thresholds. For an SDT threshold, we determined the stimulus level required to elicit spike activity in A1 that achieved a $d^{\prime}$ of 1 relative to the "no-stimulus" activity. For a JND level step, we determined the change in stimulus level required to elicit spike activity in A1 that achieved a $d$ ' of 1 relative to the "SDT threshold" activity. Because of the upper limit of our current range $(56.2 \mu \mathrm{A})$, we were unable to assess JND level steps relative to higher levels.

Evoked potential magnitudes. Evoked potentials were obtained by averaging (across 40 trials) the neural signals (analog filtered between 1.6 $\mathrm{Hz}$ and $7.5 \mathrm{kHz}$ ) recorded on each Al site for a stimulated ICC site and level. Figure 4 displays a typical example of an averaged evoked potential with the stimulus artifact present. The evoked potential consisted of a small positive deflection, followed by a larger negative deflection that then increased above baseline for tens of milliseconds. The magnitude was taken as the voltage difference between the baseline and the peak of the negative deflection. Because the baseline value could slowly shift over time before stimulus onset, we used the baseline value at stimulus onset.

Latencies. The mean first spike latency and spiking dispersion were calculated for each A1 site to ICC stimulation (for a given site and level) by selecting all the trials (of 40) that had one or more spikes, and then computing the mean and SD, respectively, of the first spike latencies across those trials. The evoked potential latency was calculated by taking the time difference between the occurrence of the negative peak of the evoked potential recorded in A1 and the ICC stimulus onset. 

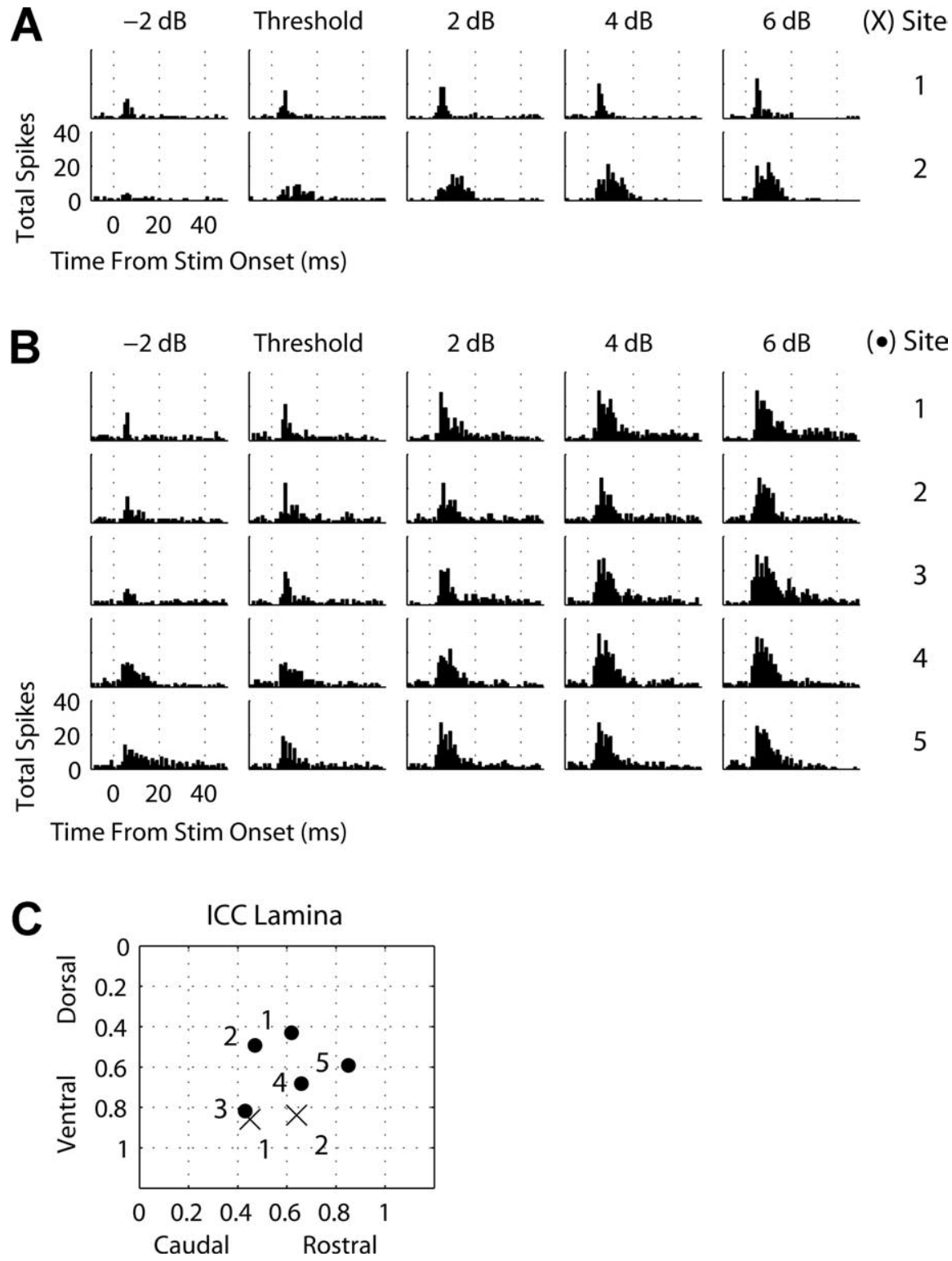

Figure 5. $\boldsymbol{A}, \boldsymbol{B}, \mathrm{PSTH}$ (1 ms bins) recorded on the main input layer site in $\mathrm{A} 1$ (same best frequency as ICC site) in response to stimulation of different ICC sites and stimulus levels relative to threshold for two animals. Stim, Stimulus. $\boldsymbol{C}$, The location of each site along a best-frequency lamina for each animal ( $\boldsymbol{A}, 12 \mathrm{kHz}$ lamina, crosses; $\boldsymbol{B}, 22 \mathrm{kHz}$ lamina, dots).

Steepest gradient axis. This is displayed in all the contour plots for the different Al parameters (i.e., threshold, evoked potential magnitude, JND level step, latency) versus ICC stimulation location, and corresponds to the linear least-squares fit of the parameter values that exhibited the steepest slope across all angles and zero reference locations (for examples, see Figs. 6-9). We then determined whether a given parameter significantly changed as a function of ICC stimulation location along that axis.

\section{Results}

All analyses are based on data from eight animals. For each animal, we electrically stimulated three to eight different sites within a single BF lamina of the ICC and recorded the corresponding neural activity in the main input layer of A1 with a similar BF. The BFs corresponding to all eight animals were $\sim 9,10,12,14,20,20$, 22 , and $23 \mathrm{kHz}$. Below, we present the effects of stimulation location along the isofrequency dimension of the ICC on temporal response patterns, thresholds, JND level steps, evoked potential magnitudes, and latencies in A1. Because of the small number of stimulation locations per animal, we grouped all the data across animals to represent a generalized isofrequency lamina. In this way, if we observe systematic trends in A1 response parameters as a function of ICC stimulation location with the grouped data, we can expect such trends for each individual lamina. However, if we do not observe any trends, we cannot exclude the possibility that grouping the data eliminated any systematic patterns caused by spatial misalignments across ICC laminas and/or location effects along the isofrequency dimension of A1. The total number of ICC locations available for analysis of each parameter varied from 28 to 44 .

\section{Temporal response patterns}

Electrical stimulation of different locations along an ICC lamina elicited different temporal response patterns on the same A1 site. Figure $5 A$ plots A1 PSTHs for varying stimulus levels relative to threshold and for two different stimulation locations within an ICC lamina $(\mathrm{BF}, 12 \mathrm{kHz})$ (Fig. $5 C$, crosses). The two sites were separated by $\sim 500 \mu \mathrm{m}$. Because of the conservative nature of our SDT threshold measure, we observed and present activity also at subthreshold levels (for justification of this method, see Materials and Methods, Data analysis, Electrical threshold). Stimulation of the caudal site (no. 1) elicited a more temporally restricted response in A1 than stimulation of the more rostral site (no. 2). Most of the spike activity for site 1 occurred within a short (few milliseconds) time window, whereas for site 2 the activity extended up to $20 \mathrm{~ms}$. This suggests that different ICC regions within the same isofrequency lamina are organized differently with respect to their output projections (including passing fibers) to higher auditory centers.

Figure $5 B$ (corresponding to Fig. $5 C$, dots) presents PSTHs from another animal and $\mathrm{BF}$ region $(22 \mathrm{kHz})$. Stimulation of site 1 , which was in the most dorsal location, appeared to elicit the most temporally restricted response at and below threshold. However, at higher stimulus levels, a more temporally diffuse activation pattern was observed. Stimulation of site 3 , which was in the most caudal region, elicited a more temporally restricted response than those of sites 4 and 5 for low levels. However, for higher stimulus levels, site 3 elicited a more temporally diffuse response and even appears to exhibit a multimodal PSTH response at $6 \mathrm{~dB}$ above threshold. Site 5 is interesting in that at subthreshold level the response is temporally scattered with a slightly elevated sustained component that lasts throughout most of the time window. However, at higher levels, this later component becomes suppressed, which is different from what is observed for sites $1-4$. This may be indicative of complex excitatory 

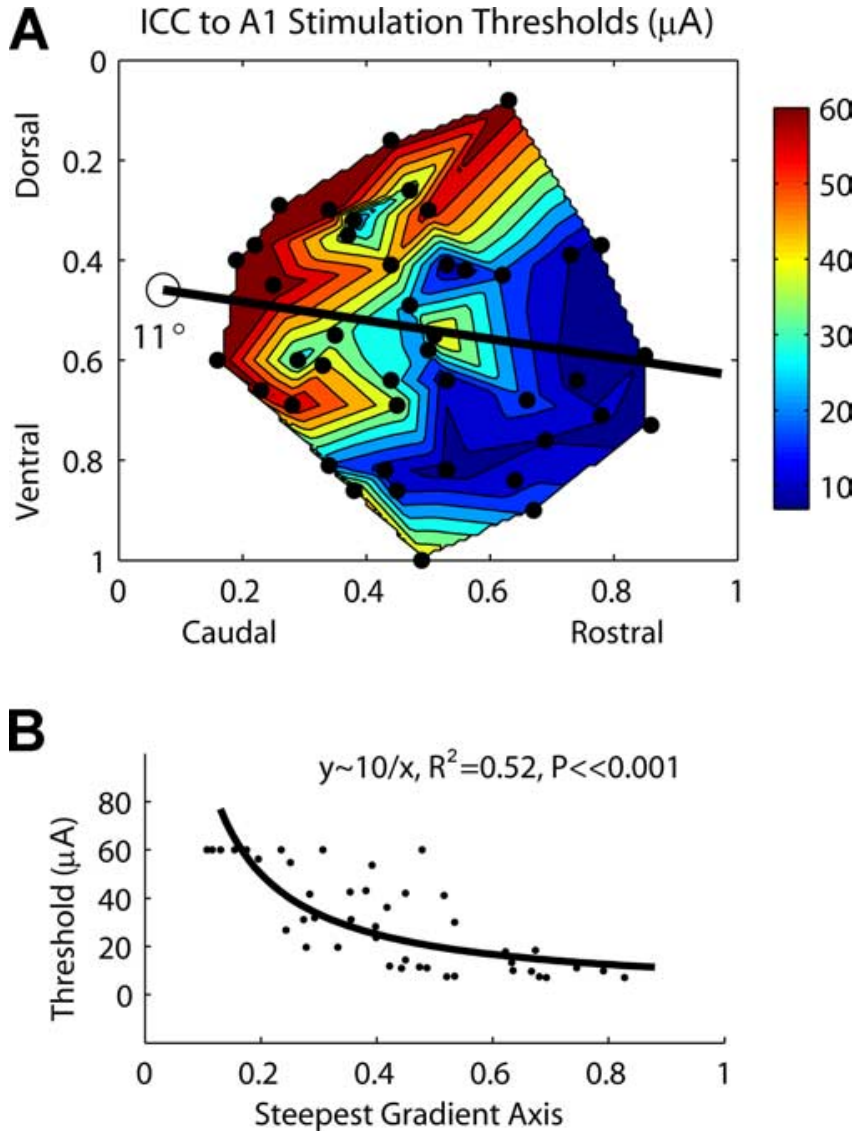

Figure 6. A, Contour plot of ICC stimulation thresholds as a function of location along the ICC laminas. The dots correspond to each of the 44 stimulation sites. For thresholds greater than our maximum level of $56.2 \mu \mathrm{A}$, we set them equal to $60 \mu \mathrm{A}$ for better visualization of the gradient. $\boldsymbol{B}$, Thresholds as a function of stimulation location along the steepest gradient axis from $\boldsymbol{A}$ (black line), which is aligned $11^{\circ}$ off the caudorostral direction. Zero (0) (along abscissa) corresponds to the location of the open circle in $\boldsymbol{A}$.

and inhibitory interactions that occur as a greater number of elements within different ICC regions are activated.

Overall, these results show that the duration and type of activity depend on location of stimulation as well as stimulus level. We did not identify any systematic stimulation location effects on these temporal features, at least with our described methods. For example, Figure $5 A$ suggests that stimulation of more caudal regions (site 1) elicit more temporally restricted activity in A1. However, stimulation of the most caudal site (no. 3) in Figure $5 B$, which was located in a similar location as site 1 from Figure $5 A$, elicited temporally restricted A1 activity only for levels near or below threshold with a broad temporal response for higher levels. It is possible that these variations in responses are associated not only with which ICC region was stimulated but also with which $\mathrm{BF}$ region or even isofrequency region within $\mathrm{A} 1$ we were recording from. It is also possible that errors caused by pooling data across animals or using multiunit responses masked any systematic location effects. Thus, a more refined method is needed to determine whether any systematic output organization of temporal features exists along the ICC laminas.

\section{Thresholds}

Location of stimulation within an isofrequency lamina of the ICC affected thresholds of activation in A1. In Figure $6 \mathrm{~A}$, one axis represents the normalized caudorostral location and the other axis represents the normalized dorsoventral location along the isofrequency laminas. The line represents the steepest gradient axis, which is aligned $11^{\circ}$ off the caudorostral direction. Generally, stimulation of more rostral and somewhat ventral ICC locations achieved lower thresholds of activation in A1. It also appears that the edge regions perpendicular to the steepest axis exhibited higher stimulation thresholds. Figure $6 B$ plots the thresholds along the steepest axis demonstrating that they significantly decrease approximately in a $1 / r$ manner $\left(R^{2}=0.52 ; p \ll\right.$ 0.001).

It is interesting that threshold differences of $>50 \mu \mathrm{A}$ could be observed by stimulating in different regions along an ICC lamina. However, most of this variation occurs for more caudal and dorsal locations. For more rostral and ventral locations, except for some of the most ventral points, threshold differences are $<10$ $\mu \mathrm{A}$. This suggests, at least in terms of functional activation of A1, that the rostral-ventral region represents a spatially distinct output region from the caudal-dorsal region. The latter consists of a steep threshold gradient that may be indicative of a less homogeneous region that systematically changes in functional organization compared with the former. It is not clear from our data whether the most ventral locations with high thresholds corresponds to a separate functional region or is part of the caudaldorsal region.

\section{Discriminable (JND) level steps}

In psychoacoustic experiments, the discrimination index, $d^{\prime}$, is used as a measure for discrimination of one stimulus condition from another. For our experiments, we used a signal detection theory framework to quantify the spike activity recorded in A1 in terms of $d^{\prime}$ to provide some interpretation of our neural responses with respect to possible perceptual effects. In particular, we were interested in quantifying the change in stimulus level (JND level step) required to elicit cortical activity corresponding to a $d^{\prime}$ value of 1 relative to threshold, and then correlate these stimulus steps as a function of location within the ICC. These results can provide insight about how perceptual level discrimination limens for AMI stimulation may change as a function of location within the ICC.

Figure $7 A$ plots the JND level steps (in microamperes) as a function of ICC stimulation location. Only 28 of the 44 sites were available for analysis because some sites required stimulus levels above our maximum level of $56 \mu \mathrm{A}$ to achieve a $d^{\prime}$ of 1 relative to threshold. Generally, stimulation of rostral ICC regions elicited smaller level steps than more caudal regions. Figure $7 B$ demonstrates that this relationship along the steepest gradient axis is significant $\left(R^{2}=0.42 ; p<0.001\right)$. The steepest gradient axis is almost parallel with the caudorostral direction ( $1^{\circ}$ off). From Figure $7 A$, it appears that the two most ventral points skew the gradient to a more parallel orientation. We also plotted JND level steps on a logarithmic scale (relative to threshold) as a function of ICC stimulation location, and did not observe any significant location trends. Plotting the level steps on a logarithmic scale equalized the values across locations. Figure $7 A$ is consistent with Figure $6 A$ in that stimulation of more rostral (and somewhat ventral) ICC regions achieves more effective activation of cortical neurons with lower current levels. Similarly, there also appears to be at least two separate regions corresponding to the caudaldorsal area and the rostral-ventral area, with possibly a third region at the ventral edge. We were unable to determine JND levels steps for higher current levels (because of the maximum current injection limit for our sites) to obtain a more complete characterization for level coding. However, our SDT threshold 


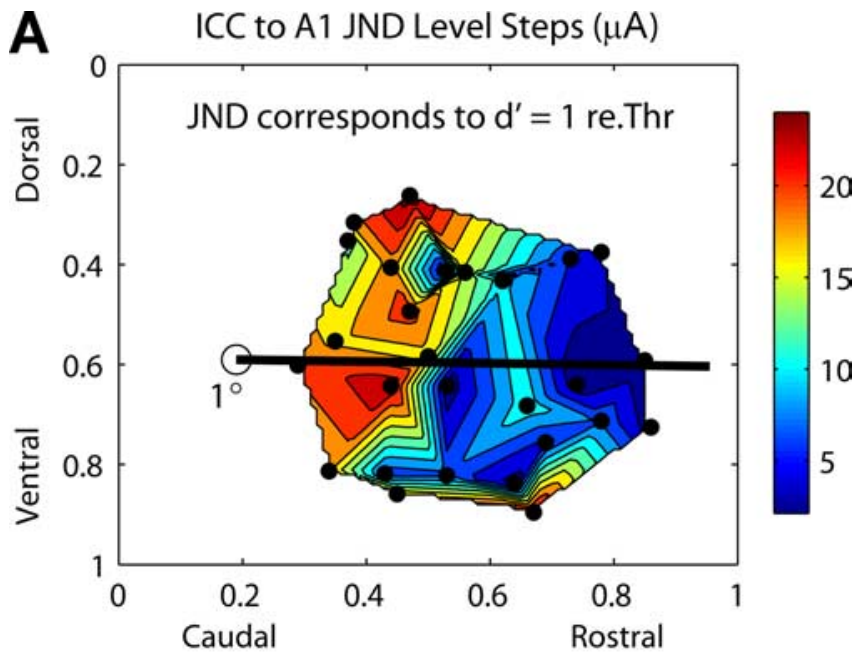

B

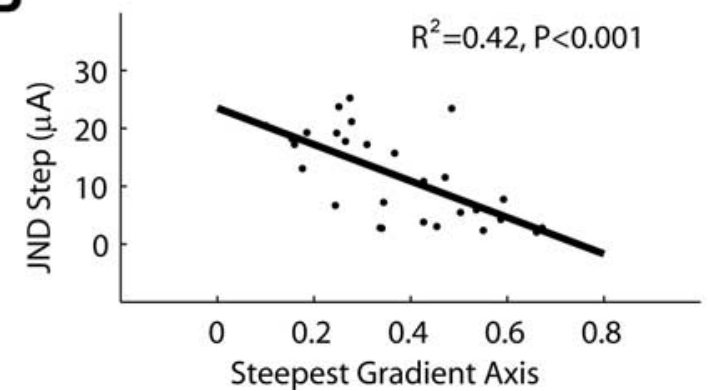

Figure 7. A, Contour plot of JND level steps (in microamperes above threshold) as a function of stimulation location along the ICC laminas. The dots correspond to each of the 28 stimulation sites. re.Thr, Relative to threshold. $\boldsymbol{B}$, JND level steps as a function of stimulation location along the steepest gradient axis from $\boldsymbol{A}$ (black line), which is aligned $1^{\circ}$ off the caudorostral direction. Zero (0) (along abscissa) corresponds to the location of the open circle in $\boldsymbol{A}$.

method was conservative, and if perceptual thresholds are much lower, then these JND level steps may correspond to moderate stimulus levels within the operating range for hearing.

\section{Evoked potential magnitudes}

Figure $8 \mathrm{~A}$ plots the evoked potential magnitudes elicited in $\mathrm{A} 1$ as a function of ICC stimulation location. A total of 36 of the 44 sites elicited detectable evoked potentials in $\mathrm{A} 1$ at our maximum level of $56.2 \mu \mathrm{A}$. The remaining eight sites were set to 0 in the plots. Generally, evoked potential magnitudes increased as more rostral ICC locations were stimulated. Figure $8 B$ demonstrates that this relationship is significant along the steepest axis approximately in a linear manner $\left(R^{2}=0.54 ; p \ll 0.001\right)$. The steepest gradient axis was aligned $6^{\circ}$ off the caudorostral direction. When Figure $8 A$ is compared with Figures $6 A$ and $7 A$, there are obvious similarities in the contour plots. Regions of higher thresholds and larger JND level steps correspond to regions of smaller evoked potentials. This is not surprising considering that, for a constant current level, ICC regions that can be more effectively activated should elicit larger evoked potentials in A1 assuming that the cortical activity is synchronized. More importantly, Figure $8 \mathrm{~A}$ confirms that a spatial distribution pattern of evoked potentials as a function of ICC stimulation location exists. This may be useful for intraoperatively identifying appropriate regions for AMI implantation (see Discussion, Implications for an AMI).
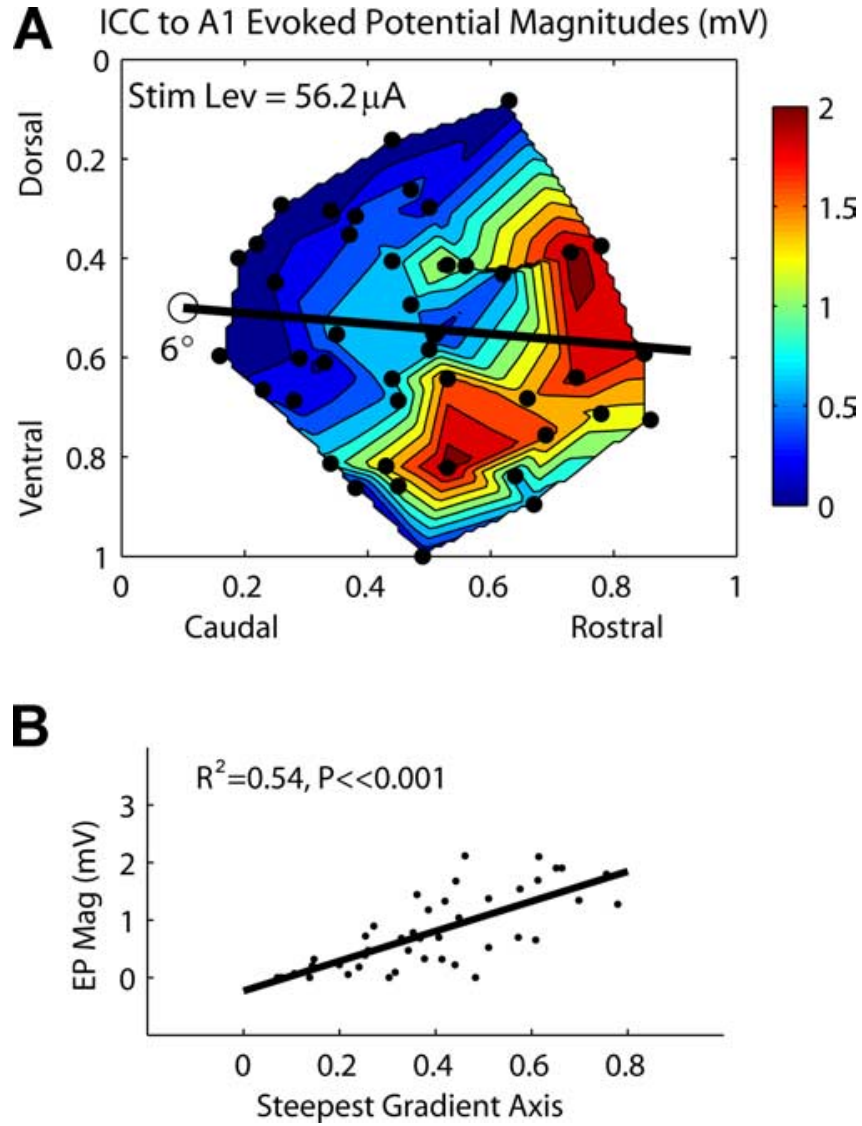

Figure 8. $\boldsymbol{A}$, Contour plot of evoked potential magnitudes recorded in $\mathrm{A} 1 \mathrm{as}$ a function of stimulation location along the ICC laminas [stimulus level (Stim Lev), 56.2 $\mu \mathrm{A}$ ]. The dots correspond to each of the 44 stimulation sites. $\boldsymbol{B}$, Evoked potential magnitudes (EP Mag) as a function of stimulation location along the steepest gradient axis from $\boldsymbol{A}$ (black line), which is aligned $6^{\circ}$ off the caudorostral direction. Zero (0) (along abscissa) corresponds to the location of the open circle in $\boldsymbol{A}$.

\section{ICC to A1 latencies}

As demonstrated in Figure 5, cortical neurons exhibited complex temporal patterns that were influenced not only by stimulation location within the ICC but also by different current levels. It was not clear from our data whether a systematic organization of temporal PSTH features, such as duration and shape, exists along an ICC lamina. However, we did observe that stimulation of certain ICC regions elicited cortical activity with different latencies. This was observed for both spike and evoked potential activity. Figure $9 \mathrm{~A}$ plots the mean first spike latencies elicited in A1 as a function of stimulation location along the ICC laminas (36 sites). For this case, the current level corresponding to SDT threshold was used. Similar trends were observed for higher current levels but with fewer data points because of our limited current range. Generally, stimulation of more caudal and dorsal regions within an ICC lamina resulted in longer cortical latencies that could be $>6 \mathrm{~ms}$ compared with other regions. This relationship was significant along the steepest gradient axis (Fig. 9B) $\left(R^{2}\right.$ $=0.23 ; p=0.003$ ) but with more variability than was observed for the other parameters. We also observed a significant relationship between mean first spike latencies and their SDs (Fig. 9C) $\left(R^{2}=0.42 ; p \ll 0.001\right)$. In other words, A1 neurons exhibited less spiking jitter (smaller SD values) for stimulation of more rostral and ventral regions where shorter latencies were observed. Approximately linear relationships between the mean and SD of 

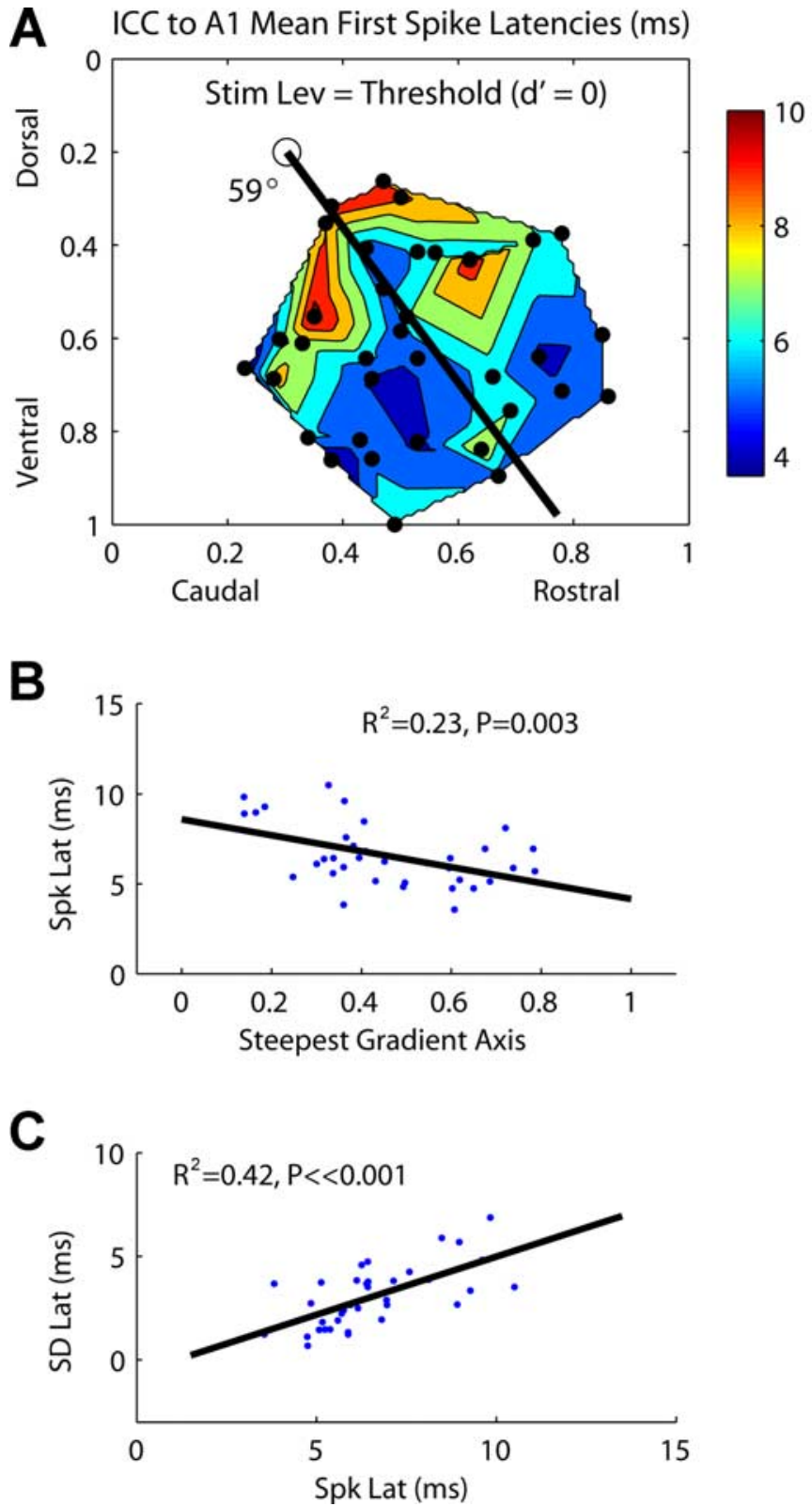
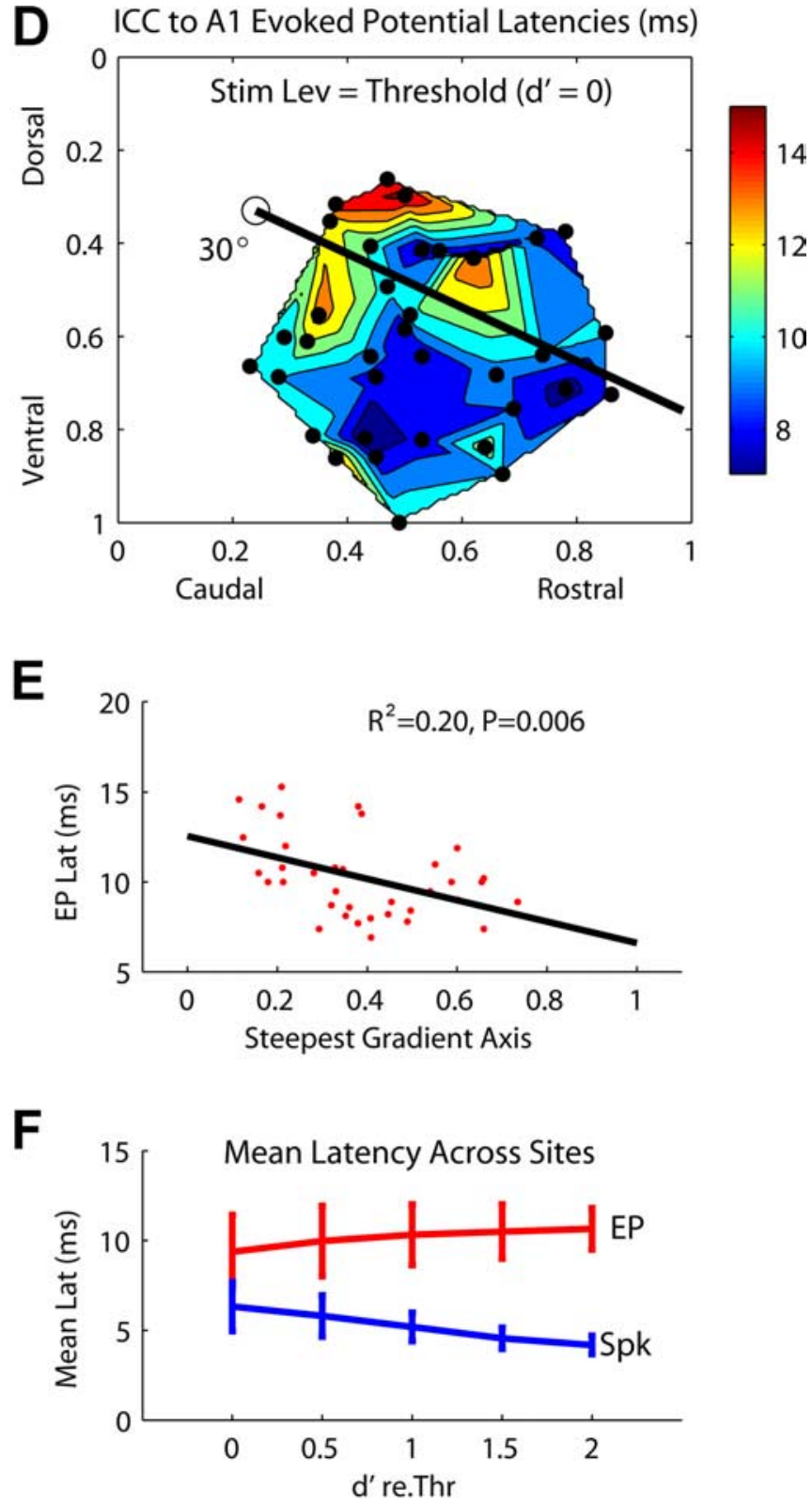

Figure 9. A, Contour plot of mean first spike latencies recorded in A1 as a function of stimulation location along the ICC laminas (at threshold level). The dots correspond to each of the 36 stimulation sites. $\boldsymbol{B}$, Mean first spike latencies as a function of stimulation location along the steepest gradient axis from $\boldsymbol{A}$ (black line), which is aligned $59^{\circ}$ off the caudorostral direction. Zero (0) (along abscissa) corresponds to the location of the open circle in $\boldsymbol{A}$. C, Relationship between mean and SD (i.e., spike jitter) of first spike latencies. $\boldsymbol{D}$, Contour plot of A1 evoked potential latencies as a function of stimulation location along the ICC laminas (at threshold level, 36 stimulation sites). $\boldsymbol{E}$, Evoked potential latencies as a function of stimulation location along the steepest gradient axis from $\boldsymbol{D}$, which is aligned $30^{\circ}$ off the caudorostral direction. $\boldsymbol{F}$, Mean and SD (across 14 sites) for the mean first spike latencies (blue) and evoked potential latencies (red) as a function of stimulus level (represented as $d^{\prime}$ ) (for more details, see Materials and Methods, Data analysis). Stim Lev, Stimulus level; Spk, spike; Lat, latency; EP, evoked potential; re.Thr, relative to threshold.

first spike latencies recorded in A1 have been observed for pure tone stimulation (Phillips and Hall, 1990; Heil, 1997).

Consistent with spike latencies, we observed that A1 evoked potential latencies were also longer for stimulation of more caudal and dorsal regions within an ICC lamina (Fig. 9D), and was significant along the steepest gradient axis (Fig. $9 E)\left(R^{2}=0.23\right.$; $p=0.003)$. The fact that $A$ and $D$ in Figure 9 are relatively similar and the evoked potential corresponds to activity from a larger population of neurons suggests that these latency differences are associated more with stimulation location within the ICC rather than properties of individual A1 neurons. The evoked potentials exhibited peak latencies that slightly increased with higher stimulus levels (larger $d^{\prime}$ values relative to threshold), whereas first spike latencies became shorter with increasing level (Fig. $9 F$ ). The mean first spike latencies were always shorter than their corresponding evoked potential latencies as expected for A1 neurons that are initially activated along the rising slope of the evoked potentials.

Although in Figure 9, $A$ and $D$, longer latencies were usually observed in more caudal and dorsal locations, compared with Figures $6-8$ the border between the caudal-dorsal region and the rostral-ventral region was less clearly defined. Furthermore, there were more discontinuous regions throughout the ICC laminas. It is possible that multiple functional zones associated with different sound features exist across a lamina and overlap with each other. 
ICC Lamina Output Regions

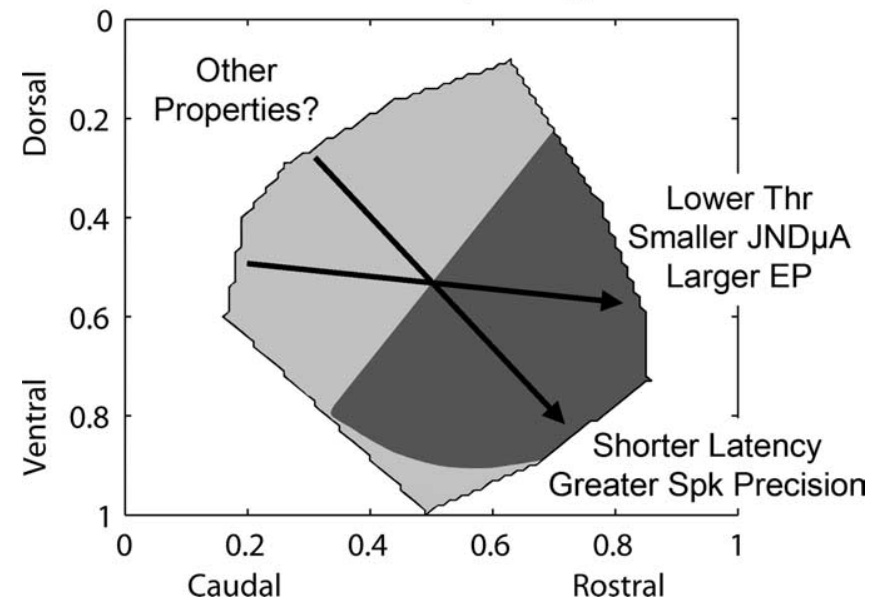

Figure 10. Summary of results describing the effects of stimulation location within an ICC lamina on different coding parameters calculated from the elicited activity in the main input layer of A1. The arrows indicate how different parameter values (labeled with text) change for stimulation along an ICC lamina that were estimated from our steepest gradient axes in Figures $6-9$. The different shaded regions (light vs dark gray) represent, in a simplified manner, two functionally distinct regions that appear to exist within an ICC lamina for output projections ascending to A1 (see Discussion, Organization within an ICC lamina). Results correspond to laminas with best frequencies between 9 and $23 \mathrm{kHz}$. EP, Evoked potential magnitude for a constant current level; JND $\mu A$, just noticeable difference level step relative to threshold in microamperes; Spk, spiking; Thr, threshold.

\section{Discussion}

The goal of this study was to assess the effects of stimulation location within an isofrequency lamina of the ICC on auditory cortical activity. A summary of our results is presented in Figure 10. Generally, stimulation of more rostral and somewhat ventral regions along an ICC lamina elicited lower thresholds, smaller JND level steps (in microamperes), and larger evoked potentials in A1. Stimulation of those regions usually elicited cortical activity with shorter latencies, which correlated with greater spiking precision. Although we observed different A1 PSTH responses (i.e., duration and shape) depending on stimulation location and level, we did not identify any systematic location effects with our current methods. More refined methods may allow us to identify other properties that are systematically organized along the ICC laminas. In a previous study (Lim and Anderson, 2006), we also observed that stimulation of more rostral ICC regions elicited greater spread of activation along the tonotopic gradient of A1. However, we only stimulated a few regions along the caudorostral dimension and still need to determine how frequencyspecific stimulation varies across an entire lamina.

\section{Organization within an ICC lamina}

Based on the contour plots from Figures 6-9, there are (at least) two separate functional regions that can be distinguished based on our A1 parameter values (excluding the few ventral edge points): a caudal-dorsal region and a rostral-ventral region. This is depicted in a simplified shading scheme in Figure 10, and corresponds to laminas with BFs between 9 and $23 \mathrm{kHz}$. The borders of the shaded regions were determined by normalizing and summing all the contour plots from Figures 6-9. Although the individual contour plots, especially those for latencies, consist of some discontinuous regions that may represent smaller functional zones within an ICC lamina, we were unable to define borders for these subregions because of our small number of data points. It is also not clear whether the most ventral region is part of the caudal-dorsal region or its own functional output zone.

Anatomical studies have shown that different brainstem nuclei project to different regions within the ICC (Roth et al., 1978; Brunso-Bechtold et al., 1981; Shneiderman and Henkel, 1987; Oliver et al., 1997; Cant and Benson, 2003; Loftus et al., 2004). For example, Loftus et al. (2004) observed in cat that, within lower frequency laminas $(<5 \mathrm{kHz})$, projections from the medial superior olivary nucleus (MSO) were more frequently located in caudal ICC regions, whereas those from the lateral superior olivary nucleus (LSO) dominated in more rostral regions. For higher frequency laminas, it appeared that inputs from the dorsal cochlear nucleus projected throughout the laminas but inputs from the LSO dominated more ventrolateral regions. These LSO inputs were further segregated in an interdigitating pattern with respect to their contralateral and ipsilateral origins (Shneiderman and Henkel, 1987). Additional evidence for a spatial organization within an ICC lamina is based on electrophysiological studies. For example, in mice it has been shown that ICC neurons with lower pure tone thresholds, sharper frequency tuning, and greater sensitivity to slower frequency sweep speeds are located more centrally within a lamina and these properties systematically change in more concentrically outward regions (Stiebler, 1986; Hage and Ehret, 2003). In cats, it has been shown that a periodotopic (best modulation frequency) map exists along the dorsomedial-to-ventrolateral dimension of the ICC laminas (Schreiner and Langner, 1988) and shorter pure-tone latencies are represented in more ventrolateral regions (Langner et al., 1987). Overall, these anatomical and electrophysiological findings support the existence of spatially distinct functional zones along the ICC laminas. However, these findings are based on projections into the ICC and it is not yet clear how these functional zones relate to output projections ascending to higher auditory structures. How does the dorsomedial-to-ventrolateral organization of monaural versus binaural inputs (and various response properties) observed in cats or the concentric functional organization of response properties identified in mice translate to the caudorostral output organization (Fig. 10) we observed in guinea pigs? Is this functional organization different across species?

In a more recent study in gerbils, Cant and Benson (2006) showed that neurons from the cochlear nuclei and nuclei of the lateral lemniscus project throughout the ICC. However, inputs from the LSO and MSO project predominantly to more rostral and lateral locations. This suggests that two distinct regions (possibly with subregions) exist within the ICC of gerbils: a caudalmedial region and a more rostral-lateral region. They further showed that these different regions project to distinct locations along the caudorostral (isofrequency) dimension of the ventral division of the medial geniculate nucleus (MGv): the caudalmedial ICC region projects to the caudal one-third of the MGv, whereas the rostral-lateral ICC region projects to the rostral twothirds of the MGv (Cant and Benson, 2007). A caudorostral organization within the MGv has also been shown in cats, in which A1 (and the anterior auditory field) receives most of its projections from the rostral MGv but few from the caudal MGv (it projects more to the posterior auditory field) (Rodrigues-Dagaeff et al., 1989). It was further shown that the caudal MGv generally exhibits longer and more widely distributed latencies, less excitatory responses, degraded synchronization properties, and systematic differences in other sound features compared with the rostral MGv. Considering that we observed longer latencies with greater spiking jitter and less activation in A1 in response to stim- 
ulation of the caudal-dorsal (i.e., caudal-medial) ICC region compared with the rostral-ventral (i.e., rostral-lateral) region, it is possible that this caudal-dorsal region in guinea pigs also projects predominantly to the caudal MGv, as observed in gerbils, and has few projections to A1, as observed in cats. This could explain the increasing gradient of thresholds observed in Figure 6 for stimulation of more caudal and dorsal regions where greater current must spread and activate the rostral-ventral ICC region to elicit activity in A1. However, the large differences in latencies ( $>6 \mathrm{~ms}$ ) between the two regions cannot be caused by current spread alone. Thus, other pathways to A1 not originating from the rostral-ventral region were likely activated. Whether this includes pathways originating from the caudal-dorsal region that do project to A1 or even onto rostral-ventral ICC neurons, or involves projections from the pericentral IC needs to be further investigated.

\section{Implications for an AMI}

Previously, we showed that ICC stimulation achieves lower thresholds, greater dynamic ranges, and more localized frequency-specific activation than cochlear stimulation (Lim and Anderson, 2003, 2006; M. Lenarz et al., 2006). These findings suggest that an AMI may provide enhanced performance over CIs. However, the complexity of the ICC along the isofrequency dimension presents a difficult challenge for determining where and how to stimulate within an ICC lamina to elicit the desired percepts. Based on our findings (Fig. 10), it may be favorable to implant an AMI in more rostral and somewhat ventral ICC regions to achieve lower neural thresholds and larger evoked potentials, assuming these neural properties correlate with lower perceptual thresholds. Stimulation of these regions also allow for smaller JND level steps (in microamperes), which could improve level coding. However, this depends on whether or not the dynamic range, which we could not obtain because of our maximum current limit, increases proportionally with JND level step size. This also depends on whether level discrimination is based on a logarithmic scale (Nelson et al., 1996; Drennan and Pfingst, 2005) for which we did not observe any significant location effects with our methods. In terms of temporal coding properties, stimulation of more rostral and ventral regions usually elicited cortical activity with shorter latencies and greater spiking precision. Considering that precise coding of temporal features of sound is important for speech perception (Shannon et al., 1995), it further supports the argument for implanting in more rostral and ventral regions. However, this argument is complicated by the fact that stimulation of more rostral ICC regions may elicit more spatially diffuse activity in A1 based on some preliminary findings (Lim and Anderson, 2006). Speech perception performance has been correlated with the ability to stimulate a greater number of frequency channels of information (Friesen et al., 2001; Shannon et al., 2004). Greater activation spread along the tonotopic gradient of A1 for ICC stimulation could reduce the number of independent frequency channels of information. Thus, stimulation of rostral ICC regions, although achieving more effective and precise neural activation, could still result in poorer performance compared with stimulation of more caudal regions. Furthermore, the inhomogeneous organization of monaural and binaural inputs throughout the ICC, as described above, may elicit varying directional percepts depending on stimulation location that could have a disruptive effect on hearing performance.

Currently, a single shank AMI with 20 electrode contacts has been developed in collaboration with Cochlear Ltd. (Lane Cove,
New South Wales, Australia) (M. Lenarz et al., 2006; T. Lenarz et al., 2006). A three-dimensional electrode array that spans multiple frequency and isofrequency locations would allow us to accommodate the different stimulation location effects. However, because of safety issues and based on technology approved for human use, a single shank array was developed and will be inserted along the tonotopic gradient of the ICC. Thus, we will have to identify an optimal isofrequency location for AMI implantation.

One approach is to implant the AMI along the tonotopic axis of the ICC but within different isofrequency locations across patients. This will allow us not only to confirm whether our location results translate to human psychophysical effects, but also to correlate stimulation location with hearing performance. If we are able to identify an optimal region for implantation and we also observe systematic location effects on evoked potential magnitudes and latencies as occurred in this study, then we may be able to use electrically evoked middle latency responses (eMLRs) to aid in implanting future patients. Realistically, the current AMI will not be reinserted after it has been implanted into the ICC. However, advancements in electrode and surgical technology, particularly applied to deep brain stimulation applications, have enabled the use of intraoperative acute recording and stimulation probes for stereotaxically identifying the target brain regions for implantation (Anderson et al., 1989; Gross et al., 2006; McCreery et al., 2006). In a similar way, we could use an acute probe to stimulate different regions within the ICC (and possibly the IC surface) and identify our target trajectory based on the magnitude and latencies of the eMLRs.

Overall, our findings demonstrate that location of stimulation along the ICC laminas affects cortical activity with respect to spectral, temporal, and intensity features, all of which have shown to be important for speech perception, and provide some insight as to where to implant the AMI in the first patients. It is still not clear how to electrically stimulate the ICC to restore intelligible speech perception and whether a single shank array will provide sufficient information for hearing restoration. However, findings across animal and cadaver studies (Lim and Anderson, 2003, 2006; M. Lenarz et al., 2006; T. Lenarz et al., 2006; Samii et al., 2007) suggest that the ICC has potential as a new site for an auditory prosthesis in NF2 patients that may outperform the ABI, and warrant additional studies and technological developments to push AMI clinical trials forward.

\section{References}

Abeles M, Goldstein Jr MH (1970) Functional architecture in cat primary auditory cortex: columnar organization and organization according to depth. J Neurophysiol 33:172-187.

Anderson DJ, Najafi K, Tanghe SJ, Evans DA, Levy KL, Hetke JF, Xue XL, Zappia JJ, Wise KD (1989) Batch-fabricated thin-film electrodes for stimulation of the central auditory system. IEEE Trans Biomed Eng 36:693-704.

Beebe X, Rose TL (1988) Charge injection limits of activated iridium oxide electrodes with $0.2 \mathrm{~ms}$ pulses in bicarbonate buffered saline. IEEE Trans Biomed Eng 35:494-495.

Britten KH, Shadlen MN, Newsome WT, Movshon JA (1992) The analysis of visual motion: a comparison of neuronal and psychophysical performance. J Neurosci 12:4745-4765.

Brunso-Bechtold JK, Thompson GC, Masterton RB (1981) HRP study of the organization of auditory afferents ascending to central nucleus of inferior colliculus in cat. J Comp Neurol 197:705-722.

Cant NB, Benson CG (2003) Parallel auditory pathways: projection patterns of the different neuronal populations in the dorsal and ventral cochlear nuclei. Brain Res Bull 60:457-474.

Cant NB, Benson CG (2006) Organization of the inferior colliculus of the gerbil (Meriones unguiculatus): differences in distribution of projections 
from the cochlear nuclei and the superior olivary complex. J Comp Neurol 495:511-528.

Cant NB, Benson CG (2007) Multiple topographically organized projections connect the central nucleus of the inferior colliculus to the ventral division of the medial geniculate nucleus in the gerbil, Meriones unguiculatus. J Comp Neurol 503:432-453.

DiCarlo JJ, Lane JW, Hsiao SS, Johnson KO (1996) Marking microelectrode penetrations with fluorescent dyes. J Neurosci Methods 64:75-81.

Drake KL, Wise KD, Farraye J, Anderson DJ, BeMent SL (1988) Performance of planar multisite microprobes in recording extracellular singleunit intracortical activity. IEEE Trans Biomed Eng 35:719-732.

Drennan WR, Pfingst BE (2005) Current-level discrimination using bipolar and monopolar electrode configurations in cochlear implants. Hear Res 202:170-179.

Ehret G (1997) The auditory midbrain, a "shunting yard" of acoustical information processing. In: The central auditory system (Ehret G, Romand R, eds), pp 259-316. New York: Oxford UP.

Friesen LM, Shannon RV, Baskent D, Wang X (2001) Speech recognition in noise as a function of the number of spectral channels: comparison of acoustic hearing and cochlear implants. J Acoust Soc Am 110:1150-1163.

Green D, Swets J (1966) Signal detection theory and psychophysics. New York: Wiley.

Gross RE, Krack P, Rodriguez-Oroz MC, Rezai AR, Benabid AL (2006) Electrophysiological mapping for the implantation of deep brain stimulators for Parkinson's disease and tremor. Mov Disord 21 [Suppl 14]:S259-S283.

Hage SR, Ehret G (2003) Mapping responses to frequency sweeps and tones in the inferior colliculus of house mice. Eur J Neurosci 18:2301-2312.

Heil P (1997) Auditory cortical onset responses revisited. I. First-spike timing. J Neurophysiol 77:2616-2641.

Huang CL, Winer JA (2000) Auditory thalamocortical projections in the cat: laminar and areal patterns of input. J Comp Neurol 427:302-331.

Langner G, Schreiner CE (1987) Topology of functional parameters in the inferior colliculus of the cat. In: New frontiers in brain research (Elsner N, Creutzfeldt OD, eds), p 122. Stuttgart: Thieme.

Langner G, Schreiner C, Merzenich MM (1987) Covariation of latency and temporal resolution in the inferior colliculus of the cat. Hear Res 31:197-201.

Lenarz M, Lim HH, Patrick JF, Anderson DJ, Lenarz T (2006) Electrophysiological validation of a human prototype auditory midbrain implant in a guinea pig model. J Assoc Res Otolaryngol 7:383-398.

Lenarz T, Moshrefi M, Matthies C, Frohne C, Lesinski-Schiedat A, Illg A, Rost U, Battmer RD, Samii M (2001) Auditory brainstem implant: part I. Auditory performance and its evolution over time. Otol Neurotol 22:823-833.

Lenarz T, Lim HH, Reuter G, Patrick JF, Lenarz M (2006) The auditory midbrain implant: a new auditory prosthesis for neural deafness- concept and device description. Otol Neurotol 27:840-845.

Lim HH, Anderson DJ (2003) Feasibility experiments for the development of a midbrain auditory prosthesis. Paper presented at First International IEEE EMBS Conference on Neural Engineering. Capri Island, Italy, March.

Lim HH, Anderson DJ (2006) Auditory cortical responses to electrical stimulation of the inferior colliculus: implications for an auditory midbrain implant. J Neurophysiol 96:975-988.

Lim HH, Anderson DJ (2007) Antidromic activation reveals tonotopically organized projections from primary auditory cortex to the central nucleus of the inferior colliculus in guinea pig. J Neurophysiol 97:1413-1427.

Loftus WC, Bishop DC, Saint Marie RL, Oliver DL (2004) Organization of binaural excitatory and inhibitory inputs to the inferior colliculus from the superior olive. J Comp Neurol 472:330-344.

Loizou PC, Dorman M, Fitzke J (2000) The effect of reduced dynamic range on speech understanding: implications for patients with cochlear implants. Ear Hear 21:25-31.

Malmierca MS, Rees A, Le Beau FE, Bjaalie JG (1995) Laminar organization of frequency-defined local axons within and between the inferior colliculi of the guinea pig. J Comp Neurol 357:124-144.

Malmierca MS, Le Beau FE, Rees A (1996) The topographical organization of descending projections from the central nucleus of the inferior colliculus in guinea pig. Hear Res 93:167-180.

McCreery D, Lossinsky A, Pikov V, Liu X (2006) Microelectrode array for chronic deep-brain microstimulation and recording. IEEE Trans Biomed Eng 53:726-737.

Merzenich MM, Reid MD (1974) Representation of the cochlea within the inferior colliculus of the cat. Brain Res 77:397-415.

Mitzdorf U (1985) Current source-density method and application in cat cerebral cortex: investigation of evoked potentials and EEG phenomena. Physiol Rev 65:37-100.

Muller-Preuss P, Mitzdorf U (1984) Functional anatomy of the inferior colliculus and the auditory cortex: current source density analyses of clickevoked potentials. Hear Res 16:133-142.

Nelson DA, Schmitz JL, Donaldson GS, Viemeister NF, Javel E (1996) Intensity discrimination as a function of stimulus level with electric stimulation. J Acoust Soc Am 100:2393-2414.

Oliver DL, Beckius GE, Bishop DC, Kuwada S (1997) Simultaneous anterograde labeling of axonal layers from lateral superior olive and dorsal cochlear nucleus in the inferior colliculus of cat. J Comp Neurol 382:215-229.

Otto SR, Brackmann DE, Hitselberger WE, Shannon RV, Kuchta J (2002) Multichannel auditory brainstem implant: update on performance in 61 patients. J Neurosurg 96:1063-1071.

Pfingst BE, Burnett PA, Sutton D (1983) Intensity discrimination with cochlear implants. J Acoust Soc Am 73:1283-1292.

Phillips DP, Hall SE (1990) Response timing constraints on the cortical representation of sound time structure. J Acoust Soc Am 88:1403-1411.

Rauschecker JP, Shannon RV (2002) Sending sound to the brain. Science 295:1025-1029.

Redies H, Sieben U, Creutzfeldt OD (1989) Functional subdivisions in the auditory cortex of the guinea pig. J Comp Neurol 282:473-488.

Rodrigues-Dagaeff C, Simm G, De Ribaupierre Y, Villa A, De Ribaupierre F, Rouiller EM (1989) Functional organization of the ventral division of the medial geniculate body of the cat: evidence for a rostro-caudal gradient of response properties and cortical projections. Hear Res 39:103-125.

Roth GL, Aitkin LM, Andersen RA, Merzenich MM (1978) Some features of the spatial organization of the central nucleus of the inferior colliculus of the cat. J Comp Neurol 182:661-680.

Samii A, Lenarz M, Majdani O, Lim HH, Samii M, Lenarz T (2007) Auditory midbrain implant: a combined approach for vestibular schwannoma surgery and device implantation. Otol Neurotol 28:31-38.

Schreiner CE, Langner G (1988) Periodicity coding in the inferior colliculus of the cat. II. Topographical organization. J Neurophysiol 60:1823-1840.

Schreiner CE, Langner G (1997) Laminar fine structure of frequency organization in auditory midbrain. Nature 388:383-386.

Semple MN, Aitkin LM (1979) Representation of sound frequency and laterality by units in central nucleus of cat inferior colliculus. J Neurophysiol 42:1626-1639.

Shannon RV, Fayad J, Moore J, Lo WW, Otto S, Nelson RA, O'Leary M (1993) Auditory brainstem implant: II. Postsurgical issues and performance. Otolaryngol Head Neck Surg 108:634-642.

Shannon RV, Zeng FG, Kamath V, Wygonski J, Ekelid M (1995) Speech recognition with primarily temporal cues. Science 270:303-304.

Shannon RV, Fu QJ, Galvin III J (2004) The number of spectral channels required for speech recognition depends on the difficulty of the listening situation. Acta Otolaryngol Suppl 552:50-54.

Shneiderman A, Henkel CK (1987) Banding of lateral superior olivary nucleus afferents in the inferior colliculus: a possible substrate for sensory integration. J Comp Neurol 266:519-534.

Skinner MW, Arndt PL, Staller SJ (2002) Nucleus 24 advanced encoder conversion study: performance versus preference. Ear Hear 23:2S-17S.

Smith PH, Populin LC (2001) Fundamental differences between the thalamocortical recipient layers of the cat auditory and visual cortices. J Comp Neurol 436:508-519.

Snyder RL, Bierer JA, Middlebrooks JC (2004) Topographic spread of inferior colliculus activation in response to acoustic and intracochlear electric stimulation. J Assoc Res Otolaryngol 5:305-322.

Stiebler I (1986) Tone-threshold mapping in the inferior colliculus of the house mouse. Neurosci Lett 65:336-340.

Wallace MN, Rutkowski RG, Palmer AR (2000) Identification and localisation of auditory areas in guinea pig cortex. Exp Brain Res 132:445-456.

Weiland JD, Anderson DJ (2000) Chronic neural stimulation with thinfilm, iridium oxide electrodes. IEEE Trans Biomed Eng 47:911-918.

Zeng FG, Galvin III JJ (1999) Amplitude mapping and phoneme recognition in cochlear implant listeners. Ear Hear 20:60-74. 